\title{
ANÁLISE CRISTALOGRÁFICA DA SOLUÇÃO SÓLIDA COM ESTRUTURA TIPO TUNGSTÊNIO BRONZE DE NIOBATO DE POTÁSSIO E ESTRÔNCIO DOPADO COM FERRO
}

\author{
Silvania Lanfredi* e Marcos A. L. Nobre \\ Departamento de Física, Química e Biologia, Faculdade de Ciências e Tecnologia, Universidade Estadual Paulista, CP 467,
} 19060-900 Presidente Prudente - SP, Brasil

Alan R. F. Lima

Departamento de Química, Universidade Estadual de Ponta Grossa, Av. Carlos Cavalcanti, 4748, Campus de Uvaranas, 84030-900 Ponta Grossa - PR, Brasil

Recebido em 27/7/09; aceito em 8/1/10; publicado na web em 5/5/10

\begin{abstract}
CRYSTALLOGRAPHIC ANALYSIS OF THE SOLID SOLUTION OF IRON DOPED POTASSIUM STRONTIUM NIOBATE WITH TETRAGONAL TUNGSTEN BRONZE STRUCTURE. Solid solution of iron doped potassium strontium niobate with $\mathrm{KSr}_{2}\left(\mathrm{FeNb}_{4}\right) \mathrm{O}_{15-\delta}$ stoichiometry was prepared by high efficiency ball milling method. Structural characterization was carried out by $\mathrm{X}$-ray diffraction. Crystalline structure was analyzed by the Rietveld refinements using the FullProf software. The results showed a tetragonal system with the tetragonal tungsten bronze structure - TTB $\left(a=12.4631\right.$ (2) $\AA$ and $\left.c=3.9322(6) \AA, \mathrm{V}=610.78(2) \AA^{3}\right)$. In this work, the sites occupancy by the $\mathrm{K}^{+}, \mathrm{Sr}^{2+}$ and $\mathrm{Fe}^{3+}$ cations on the TTB structure were determined. $\mathrm{NbO}_{6}$ polihedra distortion and its correlation with the theoretical polarization are discussed.
\end{abstract}

Keywords: $\mathrm{KSr}_{2}\left(\mathrm{FeNb}_{4}\right) \mathrm{O}_{15-\delta}$ solid solution; tetragonal tungsten bronze; Rietveld method.

\section{INTRODUÇÃO}

Óxidos ferroelétricos com estrutura tipo perovskita contendo chumbo têm sido utilizados pela indústria eletroeletrônica como atuadores, transdutores e outros componentes eletromecânicos em função de suas propriedades piezoelétricas. ${ }^{1}$ Entretanto, a toxidade do chumbo tem levado ao aumento da demanda por materiais alternativos menos agressivos ao ambiente. De forma geral, os óxidos ferroelétricos isentos de chumbo de estrutura tipo Tungstênio Bronze (TB) com simetria tetragonal possuem alta constante dielétrica, alta polarização e propriedades piezoelétricas. Estas propriedades tornam estes óxidos materiais de grande interesse tecnológico, em particular em áreas de constante expansão, como telecomunicações na região das micro-ondas, satélites e outros dispositivos relacionados.

Nos últimos anos, niobatos de metais alcalinos/alcalinos terrosos isentos de chumbo, com estrutura $\mathrm{TB}$, como $\mathrm{KSr}_{2} \mathrm{Nb}_{5} \mathrm{O}_{15}$, $\mathrm{NaSr}_{2} \mathrm{Nb}_{5} \mathrm{O}_{15}, \mathrm{KBa}_{2} \mathrm{Nb}_{5} \mathrm{O}_{15}, \mathrm{NaBa}_{2} \mathrm{Nb}_{5} \mathrm{O}_{15}, \mathrm{~K}_{3} \mathrm{Li}_{2} \mathrm{Nb}_{5} \mathrm{O}_{15}$, têm atraído interesse, principalmente pela alta anisotropia da estrutura cristalina. A alta anisotropia mantém-se até altas temperaturas nas fases centrosimétricas, não cúbicas. ${ }^{2}$ Em cerâmicas, um dos fatores que levam a essa anisotropia está associado ao crescimento anômalo dos grãos, o qual inibe a densificação e limita o número de direções de polarização. Além disso, cada grão na amostra policristalina assume uma orientação cristalográfica, diferente daquela apresentada pelos grãos vizinhos.

A estrutura TB consiste de sítios octaédricos distorcidos de $\mathrm{NbO}_{6}$ arranjados de forma a criar túneis pentagonais e tetraedrais, similares aos encontrados na estrutura perovskita, os quais são favoráveis à substituição por outros cátions e túneis trigonais vazios. ${ }^{3}$ Esta estrutura pode ser descrita pela fórmula geral $\left(\mathrm{A}^{\prime}\right)_{2}\left(\mathrm{~A}^{\prime \prime}\right)_{4}(\mathrm{C})_{4}\left(\mathrm{~B}^{\prime}\right)_{2}\left(\mathrm{~B}^{\prime \prime}\right)_{8} \mathrm{O}_{30}$, onde os sítios B' e B" são ocupados por íons nióbio, enquanto que os sítios A', A" e C podem ser ocupados por íons alcalinos, alcalinos terrosos ou chumbo. A estrutura tipo TB permite a substituição de uma grande variedade de cátions, devido à presença de vários interstícios. Em ge-

*e-mail: silvania@fct.unesp.br ral, estes compostos são dopados com cátions como $\mathrm{Li}^{+}, \mathrm{Na}^{+}, \mathrm{K}^{+}, \mathrm{Bi}^{3+}$ e $\mathrm{Ln}^{3+}$, os quais podem ocupar parcialmente os sítios A ou C. Por outro lado, o octaedro $\mathrm{BO}_{6}$ pode ser parcialmente ocupado por cátions como $\mathrm{Zr}^{4+}, \mathrm{Ti}^{4+}, \mathrm{Ta}^{5+} \mathrm{e} \mathrm{W}^{6+}$, os quais apresentam valores de raio atômico e valência próximos aos do íon $\mathrm{Nb}^{5+}$. A composição e a distribuição dos cátions em estruturas de niobatos têm uma forte influência sobre as propriedades ferroelétricas, as quais têm sido, até o momento, apenas parcialmente relacionadas às propriedades estruturais. Neste sentido, há poucas informações sobre as características estruturais e elétricas destes compostos. Uma grande parte dos esforços tem se restringido à otimização de rotas químicas, com o objetivo de garantir a estequiometria e granulometria ultrafina dos óxidos.

Este trabalho tem como objetivo a caracterização cristalográfica de uma solução sólida de $\mathrm{KSr}_{2} \mathrm{Nb}_{5} \mathrm{O}_{15}$ dopado com ferro, com estequiometria $\mathrm{KSr}_{2}\left(\mathrm{FeNb}_{4}\right) \mathrm{O}_{15-\delta}$, preparado pelo método de moagem de alta energia. Ainda, investigar a influência dos íons $\mathrm{Fe}^{3+}$ nas características estruturais da estrutura hospedeira $\mathrm{KSr}_{2} \mathrm{Nb}_{5} \mathrm{O}_{15}$. A caracterização e determinação dos parâmetros estruturais foram realizadas por difratometria de raios $\mathrm{X}$ e o refinamento da estrutura pelo método de Rietveld. A partir dos parâmetros cristalográficos foi construída a célula unitária da solução sólida de $\mathrm{KSr}_{2}\left(\mathrm{FeNb}_{4}\right) \mathrm{O}_{15-\delta}$, utilizando o programa CaRine Crystallography 3.1. ${ }^{4}$ As características estruturais foram correlacionadas à polarização ferroelétrica espontânea, bem como às propriedades dielétricas. O comportamento dielétrico da cerâmica de $\mathrm{KSr}_{2}\left(\mathrm{FeNb}_{4}\right) \mathrm{O}_{15-\delta}$ foi investigado em função da temperatura, utilizando a técnica de espectroscopia de impedância.

\section{PARTE EXPERIMENTAL}

\section{Síntese}

A solução sólida $\mathrm{KSr}_{2}\left(\mathrm{FeNb}_{4}\right) \mathrm{O}_{15-\delta}$ foi preparada utilizando-se o método de ativação mecânica por moagem de alta energia. A mistura mecânica consiste na moagem de óxidos, carbonatos ou mesmo outros sais. As características dos reagentes de partida utilizados estão listadas na Tabela 1. 
Tabela 1. Características dos reagentes de partida utilizados na preparação da solução sólida de $\mathrm{KSr}_{2}\left(\mathrm{FeNb}_{4}\right) \mathrm{O}_{15-\delta}$

\begin{tabular}{|c|c|c|c|}
\hline Nome & $\begin{array}{l}\text { Fórmula } \\
\text { Química }\end{array}$ & $\begin{array}{l}\text { Peso Molecular } \\
(\mathrm{g} / \mathrm{mol})\end{array}$ & Origem* \\
\hline Álcool isopropílico & $\left(\mathrm{CH}_{3}\right)_{2} \mathrm{CHOH}$ & 60,10 & Vetec \\
\hline Carbonato de estrôncio & $\mathrm{SrCO}_{3}$ & 147,63 & Vetec \\
\hline Carbonato de potássio & $\mathrm{K}_{2} \mathrm{CO}_{3}$ & 138,21 & Vetec \\
\hline Óxido de nióbio hidratado & $\mathrm{Nb}_{2} \mathrm{O}_{5} \cdot 4 \mathrm{H}_{2} \mathrm{O}$ & 337,82 & CBMM - Araxá \\
\hline Óxido de ferro(III) & $\mathrm{Fe}_{2} \mathrm{O}_{3}$ & 159,69 & Vetec \\
\hline
\end{tabular}

*Material P.A.

A mistura e moagem dos pós reagentes de partida foram realizadas em moinho Atritor com haste tipo Molinex, em meio de álcool isopropílico, ${ }^{5}$ a uma velocidade de $1200 \mathrm{rpm}$ durante $6 \mathrm{~h}$. Para tanto, bolas de zircônia estabilizada ( $2 \mathrm{~mm}$ ) foram utilizadas, sendo a relação em massa entre bolas e pó igual a 16:1. Após a moagem, o pó precursor foi seco em estufa (circulação forçada de ar) a $100^{\circ} \mathrm{C}$. Os efeitos dos parâmetros temperatura e tempo de tratamento térmico do pó precursor do $\mathrm{KSr}_{2}\left(\mathrm{FeNb}_{4}\right) \mathrm{O}_{15-\delta}$, em atmosfera integral de $\mathrm{O}_{2}$, foram avaliados. Pós monofásicos nanocristalinos foram obtidos após a calcinação do pó precursor a $1100{ }^{\circ} \mathrm{C}$ por $10 \mathrm{~h}$ em atmosfera de oxigênio. O pó foi desaglomerado em almofariz de ágata e passado por uma peneira (325 mesh). Em seguida, o pó foi prensado uniaxialmente na forma de pastilhas e então sinterizado a $1250^{\circ} \mathrm{C}$, em atmosfera de ar, durante 2 h com uma taxa lenta de $2{ }^{\circ} \mathrm{C} / \mathrm{min}$, obtendo-se cerâmicas densas em torno de $97 \%$ da densidade teórica, adequada à caracterização dielétrica.

\section{Caracterização dielétrica}

A caracterização dielétrica da cerâmica de $\mathrm{KSr}_{2}\left(\mathrm{FeNb}_{4}\right) \mathrm{O}_{15-\delta}$ foi realizada por espectroscopia de impedância em função da temperatura. Esta técnica tem sido aplicada no estudo das propriedades específicas dos materiais como a detecção de fenômenos correlacionados à caracterização de defeitos, transições de fases. ${ }^{6}$ A vantagem da técnica de espectroscopia de impedância é a possibilidade de caracterizar as diferentes regiões eletricamente ativas dos materiais, tanto de forma qualitativa quanto quantitativa. A permissividade complexa $\varepsilon^{*}(\omega)$ pode ser obtida a partir da impedância $\left(Z^{*}(\omega)\right)$ através da Equação $1:^{7}$

$$
\varepsilon^{*}(\omega)=1 /\left(\mathrm{j} \omega \mathrm{C}_{0} \mathrm{Z}^{*}(\omega)\right)=\varepsilon^{\prime}(\omega)-\mathrm{j} \varepsilon^{\prime \prime}(\omega)
$$

onde $\varepsilon^{\prime}(\omega)$ e $\varepsilon^{\prime \prime}(\omega)$ representam as partes real e imaginária da permissividade, respectivamente. Os valores de $\varepsilon^{\prime}(\omega)$ e $\varepsilon^{\prime \prime}(\omega)$ podem ser calculados através dos dados de impedância de acordo com as relações 2 e $3:{ }^{8}$

$$
\begin{aligned}
& \varepsilon^{\prime}(\omega)=\operatorname{Im}\left(Z^{*}\right) / Z^{2}\left(\omega \varepsilon_{0} L / A\right) \\
& \varepsilon^{\prime \prime}(\omega)=\operatorname{Re}\left(Z^{*}\right) / Z^{2}\left(\omega \varepsilon_{0} L / A\right)
\end{aligned}
$$

onde Z representa o módulo da impedância, $L$ é a espessura da amostra e $A$ é a área do eletrodo.

Um método alternativo foi utilizado para o cálculo da constante dielétrica, a partir da capacitância específica $C_{e}$. O parâmetro $C_{e}$ é obtido através da evolução do oposto da parte imaginária da impedância $-\operatorname{Im}(Z)$ em função do inverso da frequência angular $\omega$, no intervalo de altas frequências $\left(10^{5} \mathrm{a} 1,3 \times 10^{7} \mathrm{~Hz}\right) .^{9}$

A partir do valor da $C_{e}$ obtém-se a permissividade relativa através da Equação 4:

$$
\varepsilon=\frac{C_{e}}{\varepsilon_{0}} x \frac{L}{A}
$$

onde $\varepsilon_{0}$ é a permissividade do vácuo $\left(\varepsilon_{0}=8,8542 \times 10^{-12} \mathrm{~F} / \mathrm{m}\right), \mathrm{C}_{\mathrm{e}}$ é a capacitância específica, $L$ é a espessura do material e $A$ é a área do eletrodo depositado na superfície da amostra.

A caracterização dielétrica da cerâmica de $\mathrm{KSr}_{2}\left(\mathrm{FeNb}_{4}\right) \mathrm{O}_{15-\delta}$ foi realizada no intervalo de frequência de $5 \mathrm{~Hz}$ a $13 \mathrm{MHz}$, com um potencial aplicado de $500 \mathrm{mV}$, utilizando-se um analisador de impedância Alpha N High Resolution Dielectric Analyzer da Novocontrol. A amostra foi caracterizada em um porta-amostras com a configuração de dois eletrodos. A deposição dos eletrodos em faces opostas das pastilhas foi realizada com a aplicação de tinta de platina tipo TR-7905, marca Tanaka. Após secagem (temperatura ambiente durante $24 \mathrm{~h}$ ), os eletrodos foram curados a $800{ }^{\circ} \mathrm{C}$ durante $30 \mathrm{~min}$.

As medidas foram realizadas desde a temperatura ambiente até $690{ }^{\circ} \mathrm{C}$. A taxa de aquecimento foi de $2{ }^{\circ} \mathrm{C} / \mathrm{min}$. Antes de cada medida utilizou-se um tempo de estabilização térmica de $2 \mathrm{~h}$. Os valores de permissividade dielétrica da cerâmica de $\mathrm{KSr}_{2}\left(\mathrm{FeNb}_{4}\right) \mathrm{O}_{15-\delta}$ foram determinados a partir da Equação $4 .{ }^{9}$

A Figura 1 mostra as curvas de permissividade dielétrica do $\mathrm{KSr}_{2}\left(\mathrm{FeNb}_{4}\right) \mathrm{O}_{15-\delta}$ e do $\mathrm{KSr}_{2} \mathrm{Nb}_{5} \mathrm{O}_{15}$, em função da temperatura. Os valores de permissividade obtidos para o $\mathrm{KSr}_{2}\left(\mathrm{FeNb}_{4}\right) \mathrm{O}_{15-\delta}$ mostraramse superiores aos do $\mathrm{KSr}_{2} \mathrm{Nb}_{5} \mathrm{O}_{15}$ em todo intervalo de temperatura investigado. À temperatura ambiente, o valor da permissividade (2000) do $\mathrm{KSr}_{2}\left(\mathrm{FeNb}_{4}\right) \mathrm{O}_{15-\delta}$ é o dobro do valor da permissividade do $\mathrm{KSr}_{2} \mathrm{Nb}_{5} \mathrm{O}_{15}$. A curva de permissividade do $\mathrm{KSr}_{2} \mathrm{Nb}_{5} \mathrm{O}_{15}$ mostra um pico de polarização de alta intensidade em $140{ }^{\circ} \mathrm{C}$, com valor de permissividade igual a 1800 . Este ponto de máximo na permissividade tem sido associado à temperatura de Curie $\left(T_{\mathrm{C}}=157^{\circ} \mathrm{C}\right)$ do $\mathrm{KSr}_{2} \mathrm{Nb}_{5} \mathrm{O}_{15}$, ${ }^{10}$ a qual é acompanhada de transição de fase ferroelétricaparaelétrica. Ainda, dois picos assimétricos de baixa intensidade são observados na curva da permissividade do $\mathrm{KSr}_{2} \mathrm{Nb}_{5} \mathrm{O}_{15}$ ao redor de 365 e $525^{\circ} \mathrm{C}$. Em niobatos de estrutura do tipo perovskita, picos definidos ou difusos na curva da permissividade em função da temperatura têm sido atribuídos a transições de fases estruturais ou a um conjunto de transições do tipo primeira ordem. ${ }^{11}$ Aqui, a simetria tetragonal do $\mathrm{KSr}_{2} \mathrm{Nb}_{5} \mathrm{O}_{15}$ é invariante, devido à anisotropia estrutural. Portanto, transições de fases podem ocorrer via evolução de grupo espacial, a partir do grupo não centrossimétrico para um grupo centrossimétrico. Com a adição de cátions ferro na estrutura $\mathrm{KSr}_{2} \mathrm{Nb}_{5} \mathrm{O}_{15}$, este fenômeno não ocorre ao redor da temperatura de $140{ }^{\circ} \mathrm{C}$.

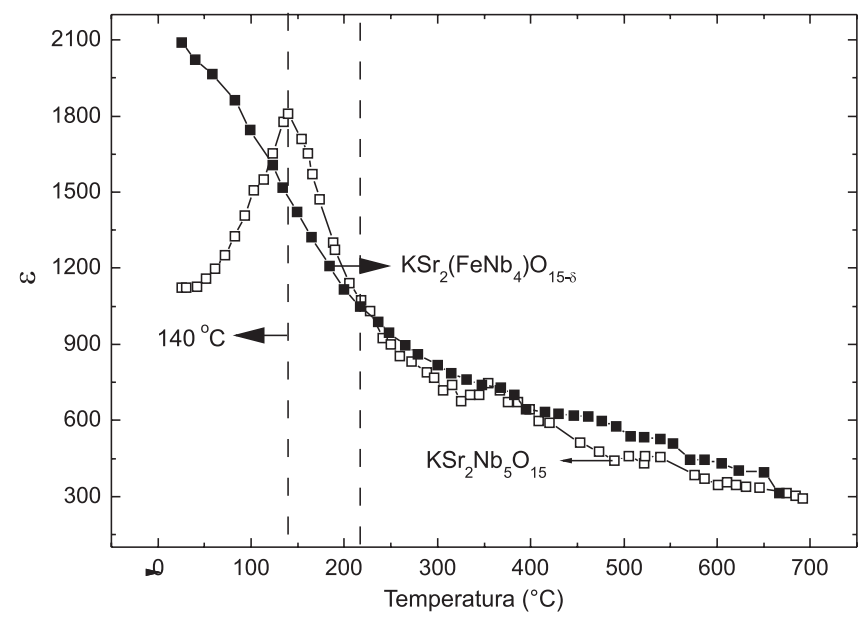

Figura 1. Curva da permissividade da cerâmica de $\mathrm{KSr}_{2}\left(\mathrm{FeNb}_{4}\right) \mathrm{O}_{15-\delta} \mathrm{em}$ função da temperatura

A Figura 2 mostra a evolução dos parâmetros $\varepsilon^{\prime}(\omega)$ e $\varepsilon^{\prime \prime}(\omega)$ obtidos a partir das Equações 2 e 3, respectivamente, com a frequência a várias temperaturas de medida. Uma dispersão na curva da permissividade real $\varepsilon^{\prime}(\omega)$, na região de baixas frequências, é observada com 
o aumento da temperatura em todo o intervalo de medida. Em geral, essas dispersões, normalmente observadas em materiais dielétricos, são associadas a um mecanismo de condução. ${ }^{7,8} \mathrm{~A}$ altas frequências, $(>1 \mathrm{kHz})$ a permissividade mostrou-se independente da frequência. Nenhum pico foi observado na curva da permissividade real $\varepsilon^{\prime}(\omega)$, em todo o intervalo de frequência investigado, o que indica ausência de fenômeno de polarização específico, como polarização de dipolos. A curva da permissividade imaginária $\varepsilon^{\prime \prime}(\omega)$ em função da frequência mostra uma diminuição de magnitude com o aumento da frequência, em todas as temperaturas de medida. Elevados valores de $\varepsilon^{\prime \prime}(\omega)$ são observados com o aumento da temperatura a baixas frequências. O grau de dispersão da permissividade imaginária diminui para frequências maiores que $1 \mathrm{kHz}$. Em todo o intervalo de frequência investigado, não foram observados picos relacionados a fenômenos de polarização nas curvas de $\varepsilon^{\prime}(\omega)$ e $\varepsilon^{\prime \prime}(\omega)$ da solução sólida $\mathrm{KSr}_{2}\left(\mathrm{FeNb}_{4}\right) \mathrm{O}_{15-\delta}$

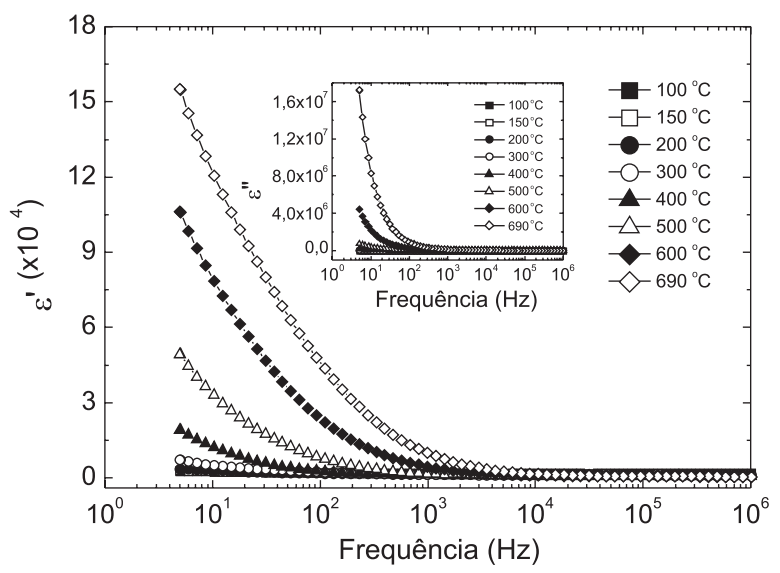

Figura 2. Evolução dos parâmetros $\varepsilon$ ' $(\omega)$ e $\varepsilon$ ” $(\omega)$ com a frequência a várias temperaturas de medida

\section{Caracterização por espectroscopia de absorção na região do infravermelho}

As ligações químicas foram analisadas por espectroscopia de absorção na região do infravermelho. As amostras foram diluídas em $\mathrm{KBr}$ na proporção 1:100. As medidas foram realizadas na região de 1000-400 $\mathrm{cm}^{-1}$, com 100 scans, usando um espectrômetro de infravermelho da marca Digilab modelo Excalibur, série FTS 3100 HE. A Figura 3 mostra os espectros de absorção na região do infravermelho para os óxidos monofásicos $\mathrm{KSr}_{2} \mathrm{Nb}_{5} \mathrm{O}_{15}$ (JCPDS: 34-0108) e $\mathrm{KSr}_{2}\left(\mathrm{FeNb}_{4}\right) \mathrm{O}_{15-\delta}$. No espectro do $\mathrm{KSr}_{2} \mathrm{Nb}_{5} \mathrm{O}_{15}$, sem a adição de ferro, identificam-se 5 bandas, sendo 3 bandas largas, assimétricas e de média intensidade nas regiões de 667, 725 e 838 $\mathrm{cm}^{-1}$ e 2 bandas mais estreitas, de baixa intensidade nas regiões de 445 e $419 \mathrm{~cm}^{-1}$. A banda de absorção presente a $838 \mathrm{~cm}^{-1}$ é atribuída ao estiramento $(\mathrm{Nb}-\mathrm{O})$ do octaedro $\mathrm{NbO}_{6}$, envolvendo a ligação do nióbio com o oxigênio apical, exibindo um alto grau de distorção. ${ }^{12}$ A substituição de $\mathrm{Nb}$ (1) por cátions ferro nos sítios octaédricos leva ao desenvolvimento de uma banda adicional de baixa intensidade em torno de $484 \mathrm{~cm}^{-1}$, associada ao modo vibracional da ligação $\mathrm{Fe}-\mathrm{O}$ do octaedro $\mathrm{FeO}_{6} \cdot{ }^{13} \mathrm{Com}$ a adição dos cátions ferro observa-se um deslocamento das bandas associadas à ligação $\mathrm{Nb}-\mathrm{O}$ para a região de menor número de onda no espectro do $\mathrm{KSr}_{2}\left(\mathrm{FeNb}_{4}\right) \mathrm{O}_{15-\delta}$.

A atribuição tentativa das bandas é listada na Tabela 2 . As bandas mais estreitas e de baixa intensidade deslocam-se para a região de 440 e $414 \mathrm{~cm}^{-1}$. Essas bandas são atribuídas ao estiramento (Nb-O) ${ }^{14}$ A banda larga e assimétrica de média intensidade na região de 725 $\mathrm{cm}^{-1}$, observada no espectro do $\mathrm{KSr}_{2} \mathrm{Nb}_{5} \mathrm{O}_{15}$, desloca-se para a região

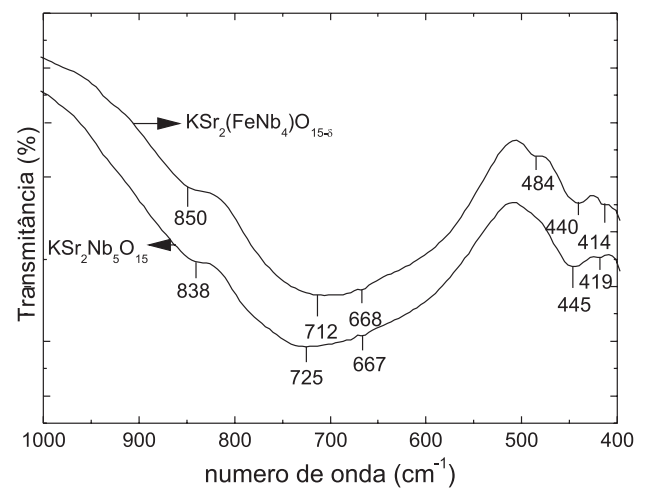

Figura 3. Espectros de absorção na região do infravermelho para o pó de $\mathrm{KSr}_{2} \mathrm{Nb}_{5} \mathrm{O}_{15}$ e do pó da solução sólida $\mathrm{KSr}_{2}\left(\mathrm{FeNb}_{4}\right) \mathrm{O}_{15-\delta}$

de $712 \mathrm{~cm}^{-1}$ no espectro do $\mathrm{KSr}_{2}\left(\mathrm{FeNb}_{4}\right) \mathrm{O}_{15-\delta}$, enquanto que a banda na região de $667 \mathrm{~cm}^{-1}$ quase não se altera com a adição de cátions ferro, sendo observada a $668 \mathrm{~cm}^{-1}$ no espectro do $\mathrm{KSr}_{2}\left(\mathrm{FeNb}_{4}\right) \mathrm{O}_{15-\delta}$. As bandas na região entre 712 e $667 \mathrm{~cm}^{-1}$ podem ser atribuídas ao estiramento simétrico ( $\mathrm{Nb}-\mathrm{O}-\mathrm{Nb}) .{ }^{15} \mathrm{O}$ deslocamento dessas bandas para a região de menor número de onda no $\mathrm{KSr}_{2}\left(\mathrm{FeNb}_{4}\right) \mathrm{O}_{15-\delta}$ implica em um aumento do caráter covalente da ligação $\mathrm{Nb}-\mathrm{O}-\mathrm{Nb} .{ }^{10} \mathrm{O}$ estiramento $(\mathrm{Nb}-\mathrm{O})$, associado à ligação do nióbio com o oxigênio apical, é observado na região de maior número de onda para o $\mathrm{KSr}_{2}\left(\mathrm{FeNb}_{4}\right)$ $\mathrm{O}_{15-\delta}\left(850 \mathrm{~cm}^{-1}\right)$, quando comparado com o $\mathrm{KSr}_{2} \mathrm{Nb}_{5} \mathrm{O}_{15}\left(838 \mathrm{~cm}^{-1}\right)$, indicando um aumento do comprimento da ligação $\mathrm{Nb}-\mathrm{O}$.

Tabela 2. Atribuição tentativa das bandas de absorção na região do infravermelho

\begin{tabular}{lccc}
\hline Atribuição & $\begin{array}{c}\text { Número de } \\
\text { onda }\left(\mathrm{cm}^{-1}\right)\end{array}$ & $\begin{array}{c}\text { Intensi- } \\
\text { dade }\end{array}$ & Ref. \\
\hline Estiramento das ligações $\mathrm{Nb}-\mathrm{O} *$ & $850-838$ & baixa & 12 \\
Estiramento das ligações $\mathrm{Fe}-\mathrm{O}$ ** & 484 & baixa & 13 \\
Estiramento das ligações $\mathrm{Nb}-\mathrm{O}$ *** & $445-419$ & baixa & 12,14 \\
Vibrações $\mathrm{Nb}-\mathrm{O}-\mathrm{Nb}$ & $712-667$ & média & 15
\end{tabular}

*Octaedros $\mathrm{NbO}_{6}$ distorcidos, ligação do $\mathrm{Nb}$ com o oxigênio apical. **Octaedro $\mathrm{FeO}_{6}$ ****Octaedros $\mathrm{NbO}_{6}$

\section{Caracterização estrutural}

A caracterização estrutural do $\mathrm{KSr}_{2}\left(\mathrm{FeNb}_{4}\right) \mathrm{O}_{15-\delta}$ foi realizada por difratometria de raios $\mathrm{X}$, utilizando um difratômetro Siemens (modelo D-5000) com radiação $\mathrm{Cu} \mathrm{K} \alpha(\lambda=1,54060)$ e um monocromador de grafite no intervalo de $5^{\circ} \leq 2 \theta \leq 80^{\circ}$. As características estruturais do $\mathrm{KSr}_{2}\left(\mathrm{FeNb}_{4}\right) \mathrm{O}_{15-\delta}$ foram analisadas através do método de Rietiveld, utilizando-se o programa FULLPROF para o refinamento dos parâmetros estruturais. ${ }^{16} \mathrm{O}$ Método de Rietveld, de refinamento de estruturas cristalinas, consiste no ajuste dos dados obtidos pela difração de raios $\mathrm{X}$ e os dados são calculados por meio de um modelo fenomenológico, pelo método dos mínimos quadrados. O principal objetivo do método de Rietveld é refinar os valores dos parâmetros estruturais da amostra em pó com os dados da difração, bem como obter os parâmetros que caracterizam a forma dos perfis dos picos de difração. O ruído de fundo foi ajustado com uma função polinomial e a forma do pico com uma função pseudo-Voigt. A dependência angular da largura do pico a meia altura $(H)$ foi definida pela função determinada por Caglioti. ${ }^{2}$ A partir das posições atômicas obtidas no refinamento foi construída a célula unitária do $\mathrm{KSr}_{2}\left(\mathrm{FeNb}_{4}\right) \mathrm{O}_{15-\delta}$, utilizando-se o programa CaRine Crystallography 3.1 ${ }^{4}$ Este programa possui uma interface gráfica, a qual permite a representação gráfica de alta resolução. Ainda, possui rotinas capazes de identificar e determinar diversos parâmetros físicos 
para materiais cristalinos, tais como sítios, número de coordenação, vacâncias, distâncias interatômicas etc.

A partir dos dados de difração de raios $\mathrm{X}$ foram também calculados o tamanho médio de cristalito e o grau de microdeformação na rede cristalina, utilizando o programa Jade 8 Plus.${ }^{17} \mathrm{O}$ tamanho médio de cristalito foi obtido pela Equação de Scherrer: ${ }^{18}$

$$
D=\frac{k \cdot \lambda}{\beta \cdot \cos \theta}
$$

onde $D$ representa o tamanho do cristalito, $\beta$ representa o alargamento do pico de difração a meia altura, $\lambda$ o comprimento de onda de raios $\mathrm{X}$ e k é uma constante que depende da simetria da reflexão e que, em geral, para pós se adota $\mathrm{k}=0,9$. $\mathrm{O}$ tamanho médio de cristalito obtido para o pó precursor do $\mathrm{KSr}_{2}\left(\mathrm{FeNb}_{4}\right) \mathrm{O}_{15-\delta}$, calcinado a $1100{ }^{\circ} \mathrm{C}$ por 10 $\mathrm{h}$, foi igual a $37 \mathrm{~nm}$. Um aumento no tamanho médio de cristalito foi observado com o aumento do tempo de tratamento térmico, acompanhado de uma pequena variação do grau de microdeformação na rede.

A microdeformação na rede foi determinada através da Equação de Williansom-Hall: ${ }^{19}$

$$
\frac{\beta \cos \theta}{\lambda}=\frac{k}{D}+\frac{4 \varepsilon}{\lambda} \cdot \operatorname{sen} \theta
$$

onde $\varepsilon$ representa a microdeformação da rede.

A evolução dos parâmetros tamanho médio de cristalito e da microdeformação na rede cristalina foi determinada para as famílias de planos cristalográficos $\{200\},\{210\},\{001\},\{330\},\{420\}$, $\{002\}$ e $\{660\}$, calculando-se o valor médio (soma do valor obtido em cada família de planos, divido pelo número total de família de planos). A microdeformação da rede, calculada para o pó precursor do $\mathrm{KSr}_{2}\left(\mathrm{FeNb}_{4}\right) \mathrm{O}_{15-\delta}$ calcinado a $1100^{\circ} \mathrm{C}$ por $10 \mathrm{~h}$, foi igual a $0,15 \mathrm{e}$ o tamanho médio de cristalito igual a $40 \mathrm{~nm}$.

\section{RESULTADOS E DISCUSSÃO}

Os parâmetros estruturais do sistema monofásico $\mathrm{KSr}_{2}\left(\mathrm{FeNb}_{4}\right) \mathrm{O}_{15-\delta}$ foram determinados utilizando-se o método de Rietveld. $\mathrm{O}$ difratograma foi indexado com base na unidade de célula tipo tetragonal. $\mathrm{O}$ refinamento foi desenvolvido considerando o grupo espacial P4bm (No 100), compatível com a regra de existência das reflexões observadas $[(O k l) k=2 n]$. Os dados cristalográficos e as condições experimentais são listados na Tabela 3. O melhor grau de refinamento para a fase $\mathrm{KSr}_{2}\left(\mathrm{FeNb}_{4}\right) \mathrm{O}_{15-\delta}$ foi obtido com os sítios pentagonal (sítio $4 \mathrm{c}$ ( $\mathrm{x}$, $\mathrm{x}+1 / 2, \mathrm{z})$ ) e tetragonal (sítio $2 \mathrm{a}(0,0, \mathrm{z})$ ) ocupados por igual quantidade de átomos de $\mathrm{K}^{+}$e $\mathrm{Sr}^{2+}$ e, para cada sítio octaedral (8d) parcialmente ocupado por átomos de $\mathrm{Fe}^{3+}$. O sítio trigonal foi considerado vazio. Os parâmetros atômicos da estrutura refinada para o pó de $\mathrm{KSr}_{2}\left(\mathrm{FeNb}_{4}\right)$ $\mathrm{O}_{15-\delta}$ são listados na Tabela 4. De acordo com os dados do refinamento obtido para o $\mathrm{KSr}_{2} \mathrm{Nb}_{5} \mathrm{O}_{15}{ }^{5}$ e para a solução sólida de $\mathrm{KSr}_{2}\left(\mathrm{FeNb}_{4}\right)$ $\mathrm{O}_{15-\delta}$, mostrados na Tabela 4, a estrutura hospedeira do $\mathrm{KSr}_{2} \mathrm{Nb}_{5} \mathrm{O}_{15}$ mostra um menor valor do parâmetro térmico isotrópico $B .{ }^{5}$ Desde que o parâmetro isotrópico está associado ao grau de ordem-desordem da estrutura, um maior valor de $B$ sugere um maior grau de desordem estrutural. Assim, por hipótese, a dopagem do $\mathrm{KSr}_{2} \mathrm{Nb}_{5} \mathrm{O}_{15}$ com ferro exibe um maior grau de desordem estática.

A Figura 4 mostra os gráficos de Rietveld observado, calculado e suas diferenças, para a solução sólida de $\mathrm{KSr}_{2}\left(\mathrm{FeNb}_{4}\right) \mathrm{O}_{15-\delta}$. Comparando-se os parâmetros de rede obtidos para o $\mathrm{KSr}_{2}\left(\mathrm{FeNb}_{4}\right) \mathrm{O}_{15-\delta}$ e para o $\mathrm{KSr}_{2} \mathrm{Nb}_{5} \mathrm{O}_{15}$ (Tabela 3), iguais a: $a=b=12,4641$ (2) $\AA, c=3,9391$ (3) Å e o volume $\mathrm{V}=611,95$ (2) $\AA^{3},{ }^{5}$ observa-se que os parâmetros de rede obtidos para a solução sólida $\mathrm{KSr}_{2}\left(\mathrm{FeNb}_{4}\right) \mathrm{O}_{15-\delta}$ mostraram-se menores, quando comparados com o sistema $\mathrm{KSr}_{2} \mathrm{Nb}_{5} \mathrm{O}_{15}$. Tal retração sugere a

\begin{tabular}{|c|c|c|}
\hline \multicolumn{3}{|l|}{ Parâmetros experimentais } \\
\hline Temperatura $\left[{ }^{\circ} \mathrm{C}\right]$ & \multicolumn{2}{|c|}{26} \\
\hline$[\mathrm{CuK \alpha}][\AA]$ & \multicolumn{2}{|c|}{1,5418} \\
\hline Monocromador & \multicolumn{2}{|c|}{ Grafite } \\
\hline Intervalo de medida (deg) & \multicolumn{2}{|c|}{$5 \leq 2 \theta \leq 80$} \\
\hline passo $\left({ }^{\circ} 2 \theta\right)$ & \multicolumn{2}{|c|}{0,02} \\
\hline \multicolumn{3}{|c|}{ Dados cristalográficos } \\
\hline \multirow{3}{*}{$\begin{array}{l}\text { Fórmula } \\
\text { Simetria } \\
\text { Grupo Espacial }\end{array}$} & $\mathrm{KSr}_{2} \mathrm{Nb}_{5} \mathrm{O}_{15}$ & $\begin{array}{l}\mathrm{KSr}_{2}\left(\mathrm{FeNb}_{4}\right) \\
\mathrm{O}_{15-\delta}\end{array}$ \\
\hline & Tetragonal & Tetragonal \\
\hline & $\mathrm{P} 4 \mathrm{bm}\left(\mathrm{n}^{\circ} 100\right)$ & P4bm(nº100) \\
\hline$a[\AA]$ & 12,4641 & 12,4631 \\
\hline$c[\AA]$ & 3,9391 & 3,9322 \\
\hline $\mathrm{V}[\AA ̊]$ & 611,95 & 610,78 \\
\hline Z & 2 & 2 \\
\hline
\end{tabular}

Tabela 3. Parâmetros experimentais e os dados cristalográficos

Tabela 4. Coordenadas atômicas (x, y, z), parâmetro térmico isotrópico $B$, ocupação relativa $P$ para a solução sólida $\mathrm{KSr}_{2}\left(\mathrm{FeNb}_{4}\right) \mathrm{O}_{15-\delta}$

\begin{tabular}{lcccccc}
\hline Átomo & $\begin{array}{c}\text { Posição de } \\
\text { Wyckoff }\end{array}$ & $\mathrm{x}$ & $\mathrm{y}$ & $\mathrm{z}$ & $\mathrm{B}$ & $P$ \\
\hline $\mathrm{Sr}(1)$ & $2 a$ & 0 & 0 & 0 & $1,36(1)$ & 0,2 \\
$\mathrm{~K}(1)$ & $2 a$ & 0 & 0 & 0 & $1,36(1)$ & 0,2 \\
$\mathrm{~K}(2)$ & $4 c$ & 0,16674 & 0,66674 & 0,00050 & $2,70(3)$ & 0,2 \\
$\mathrm{Sr}(2)$ & $4 c$ & 0,16674 & 0,66674 & 0,00050 & $2,70(3)$ & 0,2 \\
$\mathrm{Fe}(1)$ & $8 d$ & 0 & $1 / 2$ & $1 / 2$ & $2,05(5)$ & 0,25 \\
$\mathrm{Nb}(2)$ & $8 d$ & 0,07580 & 0,21152 & 0,49800 & $0,23(9)$ & 1 \\
$\mathrm{O}(1)$ & $8 d$ & 0,16443 & 0,09831 & $1 / 2$ & $3,07(2)$ & 1 \\
$\mathrm{O}(2)$ & $8 d$ & 0,34073 & 0,00598 & 0,46600 & $5,27(2)$ & 1 \\
$\mathrm{O}(3)$ & $8 d$ & 0,07683 & 0,22013 & 0,02600 & $2,14(5)$ & 1 \\
$\mathrm{O}(4)$ & $4 c$ & 0,27363 & 0,77363 & $1 / 2$ & $1,17(7)$ & 0,5 \\
$\mathrm{O}(5)$ & $2 b$ & 0 & $1 / 2$ & 0,02700 & $2,74(9)$ & 0,25 \\
\hline
\end{tabular}

atuação de dois fatores principais. O primeiro, refere-se à substituição parcial dos íons $\mathrm{Nb}^{5+}\left(r_{N b}^{+5}=69 \mathrm{pm}\right)$ pelos íons $\mathrm{Fe}^{3+}$ na rede cristalina, o qual possui um raio iônico menor $\left(r_{\mathrm{Fe}}^{+3}=67 \mathrm{pm}\right)$. O segundo fator pode ser relacionado ao menor comprimento médio nas ligações $\mathrm{Fe}-\mathrm{O}(1,99$ $\AA$ ), quando comparado com as ligações de $\mathrm{Nb}-\mathrm{O}(2,01 \AA) .{ }^{10}$

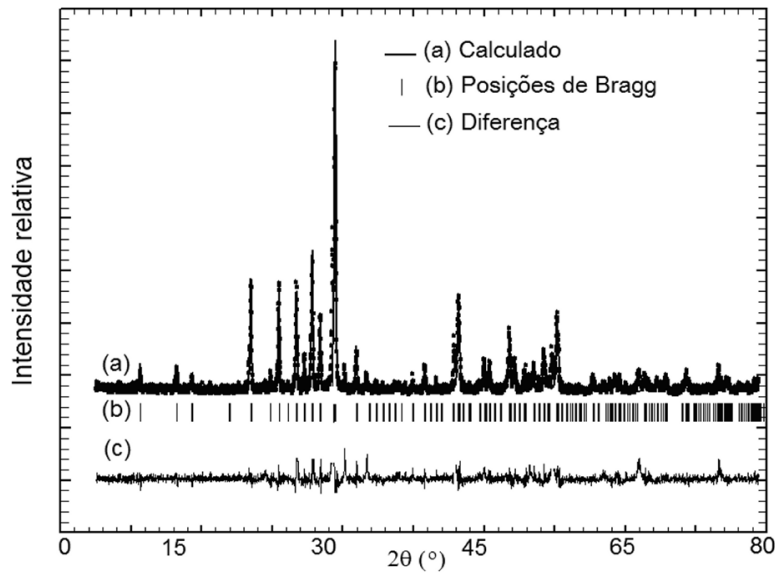

Figura 4. Gráfico de Rietveld para o pó da solução sólida $\mathrm{KSr}_{2}\left(\mathrm{FeNb}_{4}\right) \mathrm{O}_{15-\delta}$ preparado por moagem de alta energia

A partir dos dados de ocupação relativa $(P)$, coordenadas atômicas (x, y, z) (Tabela 4), parâmetros de rede e grupo espacial, foi possível simular 
a estrutura cristalográfica do $\mathrm{KSr}_{2}\left(\mathrm{FeNb}_{4}\right) \mathrm{O}_{15-\delta}$ calcinado a $1110^{\circ} \mathrm{C}$ por $10 \mathrm{~h}$, utilizando-se o programa CaRIne Crystallography $3.1^{\circledR},{ }^{4}$ Figura 5.

A Figura 5 mostra a representação gráfica da célula unitária obtida para o pó da solução sólida $\mathrm{KSr}_{2}\left(\mathrm{FeNb}_{4}\right) \mathrm{O}_{15-\delta}$, a partir dos dados listados na Tabela 4. Os átomos de nióbio e ferro são coordenados por átomos de oxigênio, 1:6, sendo que quatro átomos de oxigênio encontram-se, a priori, no mesmo plano do átomo de nióbio e ferro, e os outros dois acima e abaixo do plano, respectivamente. Essa é a condição favorável para a formação de sítios do tipo $\mathrm{M}$ (octaédricos) na estrutura. ${ }^{10}$ Ainda, na representação da Figura 5 não são mostrados os oxigênios do plano y (plano de cima).

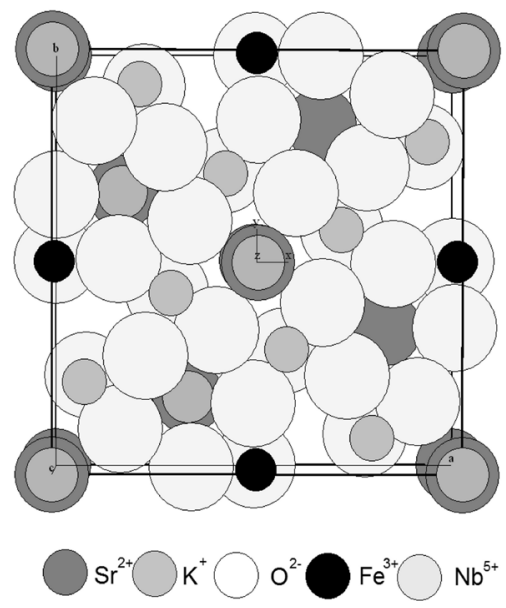

Figura 5. Representação da célula unitária obtida em função dos parâmetros ocupação relativa $(P)$, coordenadas atômicas $(x, y, z)$, parâmetros de rede $e$ grupo espacial, listados na Tabela 4, para a solução sólida $\mathrm{KSr}_{2}\left(\mathrm{FeNb}_{4}\right)$ $O_{15-\delta}$ calcinada a $1110^{\circ} \mathrm{C}$ por $10 \mathrm{~h}$

A Figura 6 mostra a representação esquemática da estrutura do $\mathrm{KSr}_{2}\left(\mathrm{FeNb}_{4}\right) \mathrm{O}_{15-\delta}$, explicitando-se os poliedros de coordenação, gerada via o programa CaRIne Crystallography $3.1^{\circledR}$, utilizando-se os dados da Tabela 4. Na representação adotada um átomo de oxigênio $\mathrm{O}^{2-}$ do plano z é omitido. De acordo com a representação da célula unitária para o $\mathrm{KSr}_{2}\left(\mathrm{FeNb}_{4}\right) \mathrm{O}_{15-\delta}$, os sítios pentagonais e tetragonais são ocupados por igual quantidade de átomos de $\mathrm{K}^{+} \mathrm{e} \mathrm{Sr}^{2+}$. Os resultados são compatíveis com aqueles reportados previamente. ${ }^{3}$ A ocupação dos sítios octaedrais pelos íons de $\mathrm{Fe}^{3+}$ fica evidente quando se analisa a Figura 6, os átomos $\mathrm{de}^{3+} \mathrm{F}^{3+}$ são coordenados por 6 átomos de $\mathrm{O}^{2-}$, formando octaedros $\left[\mathrm{FeO}_{6}\right]$. Ambos os poliedros são distorcidos, sendo possível identificar uma rotação ao longo do eixo c, o plano central mostra ligações $\mathrm{Nb}-\mathrm{O}(2)$, $\mathrm{Nb}-\mathrm{O}(3), \mathrm{Nb}-\mathrm{O}(4), \mathrm{Nb}-\mathrm{O}(5)$ com comprimentos alterados.

A Tabela 5 lista as distâncias interatômicas das ligações $\mathrm{Nb}-\mathrm{O}$ e Fe-O nos sítios octaédricos do $\mathrm{KSr}_{2}\left(\mathrm{FeNb}_{4}\right) \mathrm{O}_{15-\delta}$. A representação esquemática dos octaedros de $\left[\mathrm{FeO}_{6}\right]$ e $\left[\mathrm{NbO}_{6}\right]$ é mostrada nas Figura $7 \mathrm{a}$ e $7 \mathrm{~b}$, respectivamente.

De acordo com a Tabela 5, como esperado, identifica-se um menor comprimento das ligações $\mathrm{Fe}-\mathrm{O}$ comparadas às ligações $\mathrm{Nb}-\mathrm{O}$. Assim, os octaedros $\left[\mathrm{FeO}_{6}\right]$ possuem um volume menor que aquele dos octaedros $\left[\mathrm{NbO}_{6}\right]$. Isto resulta em uma diminuição do volume da célula unitária, comparando-se com a estrutura do $\mathrm{KSr}_{2} \mathrm{Nb}_{5} \mathrm{O}_{15}$, Tabela 3. De acordo com a Figura 7, os poliedros $\left[\mathrm{FeO}_{6}\right]$ e $\left[\mathrm{NbO}_{6}\right]$ mostram uma distorção do tipo elongação, sendo que tal elongação se mostra assimétrica e caracterizada pelo deslocamento do oxigênio apical. Ainda, o octaedro $\left[\mathrm{NbO}_{6}\right]$ mostra um aumento do comprimento da ligação $\mathrm{Nb}-\mathrm{O}$ (6) (ligação correspondente ao oxigênio (6) do octaedro ligado ao nióbio, Figura $7 \mathrm{~b}$ ), indicando uma rotação sobre o eixo apical do octaedro. Como um todo, os graus de distorção do

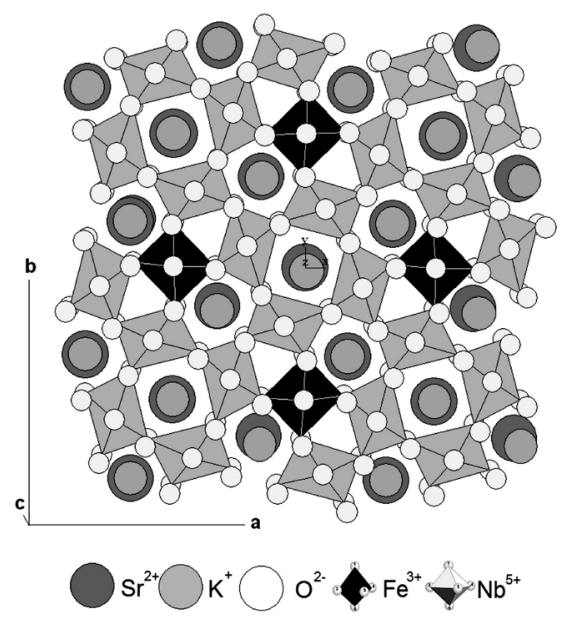

Figura 6. Representação esquemática da estrutura tungstênio bronze de simetria tetragonal do $\mathrm{KSr}_{2}\left(\mathrm{FeNb}_{4}\right) \mathrm{O}_{15-\delta}$ do ponto de vista perpendicular ao plano ab. Em destaque os octaedros associados à coordenação de $\mathrm{Nb}(2) \mathrm{e}$ Fe pelos oxigênios

Tabela 5. Distâncias interatômicas das ligações Nb-O e Fe-O nos sítios octaédricos do $\mathrm{KSr}_{2}\left(\mathrm{FeNb}_{4}\right) \mathrm{O}_{15-\delta}$

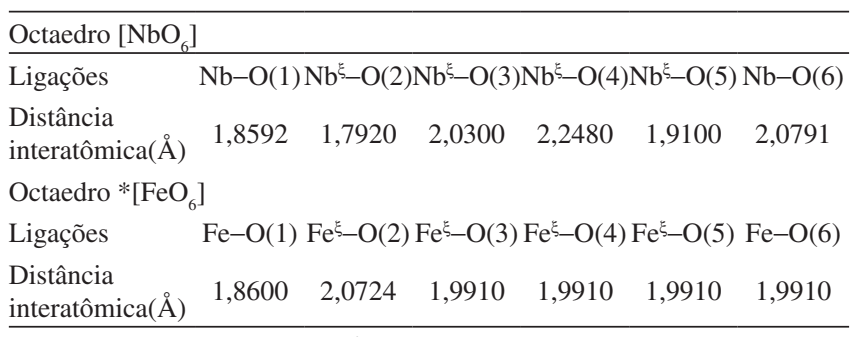

*Fe: substituindo parte do $\mathrm{Nb}$. $\xi$ átomos do plano central do octaedro

[FeO6] e [NbO6] são compatíveis com o desenvolvimento de rotação e inclinação dos poliedros. Ambos comprimentos de ligações entre nióbio e oxigênios apicais (O1 e O6) são diferentes, o que pode ser associado à posição do nióbio fora do centro.

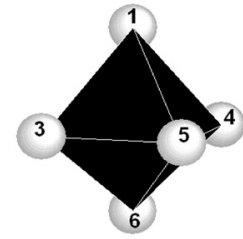

(a)

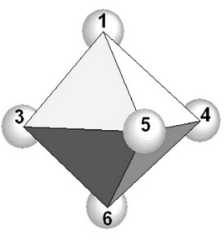

(b)
Figura 7. Representação esquemática de dois sítios octaédricos vizinhos no plano ab: (a) sítio octaédrico de $\mathrm{FeO}_{6}$ e (b) sítio octaédrico de $\mathrm{NbO}_{6}$

A polarização ferroelétrica em óxidos niobatos ferroelétricos é atribuída ao deslocamento do $\mathrm{Nb}$ a partir da posição central do octaedro $\left[\mathrm{NbO}_{6}\right]$, off-center, $\Delta \mathrm{z}$. O valor de z (coordenada de $\mathrm{Nb}$ (2) em sítios $8 j$ ) é diferente de $z=1 / 2$. A subtração dos dois comprimentos (Nb-O6) e (Nb-O1) é proporcional ao dobro do deslocamento do nióbio a partir do centro do poliedro, $\Delta \mathrm{z}$, ver discussão da Figura 7. O caráter off-center do nióbio conduz a um alto grau de polarização, o qual justifica a alta permissividade (2000) à temperatura ambiente, como mostrado na Figura 1. É interessante notar que o cátion ferro contribui para a polarizabilidade estrutural, em específico para a polarização estrutural em função da característica off-center. Ainda, o alto caráter covalente da ligação $\mathrm{Nb}-\mathrm{O}$ garante uma interação dipolar de longo alcance dentro dos domínios ferroelétricos, levando ao 
clássico comportamento ferroelétrico. A polarização espontânea, $\mathrm{P}_{\mathrm{E}}$, do [NbO6] depende da magnitude do deslocamento $\Delta \mathrm{z}$. A magnitude do parâmetro $\mathrm{P}_{\mathrm{E}}$ pode ser determinada pela Equação $7:{ }^{20}$

$$
\mathrm{P}_{\mathrm{E}}=(258 \pm 9) \Delta \mathrm{z} \mu \mathrm{C} \mathrm{cm} \mathrm{cm}^{-2}
$$

onde $\Delta z$ é igual a $0,1099 \AA$.

A polarização espontânea na estrutura do $\mathrm{KSr}_{2}\left(\mathrm{FeNb}_{4}\right) \mathrm{O}_{15-\delta}$, calculada pela Equação 7 é igual $28,35 \mu \mathrm{C} \mathrm{cm}^{-2}$. Este valor é mais de dez vezes maior que o valor obtido para a polarização espontânea dos octaedros de [NbO6] da estrutura do $\mathrm{KSr}_{2} \mathrm{Nb}_{5} \mathrm{O}_{15}$, o qual é em torno de $2,46 \mu \mathrm{C} \mathrm{cm}^{-2} .{ }^{21}$ Os valores obtidos para o $\mathrm{KSr}_{2}\left(\mathrm{FeNb}_{4}\right) \mathrm{O}_{15-\delta}$ são compatíveis com aqueles obtidos para compostos ferroelétricos de estrutura TB com grupo espacial P4bm, como o $\mathrm{Ba}_{5 / 4} \mathrm{Sr}_{15 / 4} \mathrm{Nb}_{10} \mathrm{O}_{30}$ e o $\mathrm{Ba}_{6} \mathrm{Ti}_{2} \mathrm{Nb}_{8} \mathrm{O}_{30}$ os quais apresentam valores de polarização espontânea em torno de $25 \mu \mathrm{C} \mathrm{cm}^{-2} .{ }^{20}$

\section{CONCLUSÕES}

A técnica de moagem de alta energia mostrou-se adequada à preparação de pós monofásicos e cristalinos da solução sólida $\mathrm{KSr}_{2}\left(\mathrm{FeNb}_{4}\right) \mathrm{O}_{15-\delta}$. A caracterização estrutural mostrou que a substituição de $1 / 5$ dos átomos de nióbio por $\mathrm{Fe}^{3+}$ na estrutura do $\mathrm{KSr}_{2} \mathrm{Nb}_{5} \mathrm{O}_{15}$ resulta em uma diminuição dos parâmetros de rede. A determinação dos parâmetros cristalográficos mostra que os sítios tetragonais e pentagonais são ocupados por iguais quantidades de íons $\mathrm{K}^{+}$e $\mathrm{Sr}^{+2}$ e os sítios octaédricos pelos íons $\mathrm{Fe}^{3+} \mathrm{e} \mathrm{Nb}^{5+}$. Em específico, o $\mathrm{Fe}^{3+}$ ocupa posições de $\mathrm{Nb}(1)$. Tal ocupação induz a uma intensa distorção dos poliedros $\left[\mathrm{NbO}_{6}\right]$, os quais sofrem rotação e inclinação. A distância dos cátions $\mathrm{Nb}$ do centro do poliedro aumenta com a adição dos íons $\mathrm{Fe}^{3+}$, o que aumenta o grau de polarização da rede cristalina. Este comportamento pode ser confirmado nas curvas de permissividade, com o aumento dos valores de $\varepsilon$ com a substituição de cátions nióbio por cátion ferro. A transição de fase ferroelétrica $(\mathrm{P} 4 \mathrm{bm}) \rightarrow$ paraelétrica $(\mathrm{P} 4 / \mathrm{mbm})$ é suprimida.

\section{AGRADECIMENTOS}

Ao apoio financeiro da FAPESP (Processo: 07/03510-9) e CNPq (Processo: 474627/2004-7).

\section{REFERÊNCIAS}

1. Guerra, J. de los S.; Vernet, M.; Garcia, D.; Eiras, J. A.; Appl. Phys. Lett. 2007, 91, 062951.

2. Simon, A.; Ravez, J.; C. R. Chim. 2006, 9, 1268.

3. Lanfredi, S.; Cardoso, C. X.; Nobre, M. A. L.; Mater. Sci. Eng., B 2004, $112,139$.

4. Boudais, C.; Monceau, D.; CARINE CRYSTALLOGRAPHY 3.1 (R $^{\text {; The }}$ Crystallographic Software for Research and Teaching; France, 1998.

5. Lanfredi, S.; Trindade, L. R.; Barros, A. R.; Feitosa, N. R.; Nobre, M. A. L.; Cerâmica 2005, 51, 151.

6. Nobre, M. A. L.; Lanfredi, S.; Appl. Phys. Lett. 2003, 82, 2284.

7. Nobre, M. A. L.; Lanfredi, S.; J. Phys. Chem. Solids 2003, 64, 2457.

8. Nobre, M. A. L.; Lanfredi, S.; Catal. Today 2003, 78, 529.

9. Nobre, M. A. L.; Lanfredi, S.; J. Phys. Chem. Solids 2001, 62, 1999.

10. Belghiti, H. El. A.; Simon, A.; Gravereau, P.; Villesuzanne, A.; Elaatmani, M.; Ravez, J.; Sol. State Sci. 2002, 4, 933.

11. Nobre, M. A. L.; Lanfredi, S.; J. Phys. Condens. Matter, 2000, 12, 7833.

12. Cordier, S.; Hernandez, O.; Thépot, J. Y.; Shames, A. I.; Perrin, C.; Inorg. Chem. 2003, 42, 1101.

13. Rusu, D.; Ardelean, I.; Mater. Res. Bull. 2008, 43, 1724.

14. Farrel, F. I.; Maroni, V. A.; Spiro, T. G.; Inorg. Chem. 1969, 8, 2638.

15. Lanfredi, S.; Folgueras-Domínguez, S.; Rodrigues, A. C. M.; J. Mater Chem. 1995, 5, 1957.

16. Carvajal, J. R.; FULLPROFF 2000; Program for Crystal Structure Refinement; Laboratoire Léon Brillouin, CEA/Saclay, France, 2008.

17. JADE 8 PLUS, XRD Pattern Processing and Identification Program, Materials Data, Inc. 1995-2007.

18. Azároff, L. V.; Buerguer, M. J.; The Powder Method in X-Ray Crystallography, McGraw-Hill: New York, 1958.

19. Williansom, G. K.; Hall, W. H.; Acta Metall. 1953, 1, 22.

20. Abrahams, S. C. ; Kurtz, S. K.; Jamieson, P. B.; Phys. Rev. 1968, 172, 551.

21. Alkoyw, S.; Duran, C. J.; Am. Ceram. Soc. 2008, 91,1597. 